Journal of Bioinformatics and Computational Biology

Vol. 14, No. 5 (2016) 1644003 (16 pages)

(C) The Author(s)

DOI: $10.1142 / \mathrm{S} 0219720016440030$

\title{
KDSNP: A kernel-based approach to detecting high-order SNP interactions
}

\author{
Kento Kodama* and Hiroto Saigo ${ }^{\dagger}$ \\ Department of Bioscience and Bioinformatics \\ Kyushu Institute of Technology \\ 680-4 Kawazu, Iizuka 820-8502, Fukuoka, Japan \\ *n673210k@mail.kyutech.jp \\ †saigo@bio.kyutech.ac.jp
}

Accepted 24 August 2016

Published 24 October 2016

\begin{abstract}
Despite the accumulation of quantitative trait loci (QTL) data in many complex human diseases, most of current approaches that have attempted to relate genotype to phenotype have achieved limited success, and genetic factors of many common diseases are yet remained to be elucidated. One of the reasons that makes this problem complex is the existence of single nucleotide polymorphism (SNP) interaction, or epistasis. Due to excessive amount of computation for searching the combinatorial space, existing approaches cannot fully incorporate highorder SNP interactions into their models, but limit themselves to detecting only lower-order SNP interactions. We present an empirical approach based on ridge regression with polynomial kernels and model selection technique for determining the true degree of epistasis among SNPs. Computer experiments in simulated data show the ability of the proposed method to correctly predict the number of interacting SNPs provided that the number of samples is large enough relative to the number of SNPs. For cases in which the number of the available samples is limited, we propose to perform sliding window approach to ensure sufficiently large sample/SNP ratio in each window. In computational experiments using heterogeneous stock mice data, our approach has successfully detected subregions that harbor known causal SNPs. Our analysis further suggests the existence of additional candidate causal SNPs interacting to each other in the neighborhood of the known causal gene. Software is available from https://github.com/ HirotoSaigo/KDSNP.
\end{abstract}

Keywords: Epistasis; gene-gene interaction; GWAS; kernel ridge regression; polynomial kernel.

\section{Introduction}

With the recent advances in high-throughput genotyping technologies, hundreds of thousands of single nucleotide polymorphisms (SNP) are assayed per person to

${ }^{\dagger}$ Current address: Faculty of Information Science and Electrical Engineering, Kyushu University, 744 Motooka, Nishi-ku, Fukuoka 819-0395, Japan.

This is an Open Access article published by World Scientific Publishing Company. It is distributed under the terms of the Creative Commons Attribution 4.0 (CC-BY) License. Further distribution of this work is permitted, provided the original work is properly cited. 
identify and characterize relationship between genotypes and clinical measurements. Despite the accumulation of genome wise association study (GWAS) data of this kind, current approaches have not been always successful in explaining the relationships between genotypes and phenotypes for many common complex multifactorial human traits. ${ }^{1}$ There are two major explanations believed to be the reason for this limited success. The first one is "rare variants-common diseases" hypothesis, which attributes the reason to the lack of sequencing sensitivity and doubts the existence of very rare causal SNPs those are not sequenced at the current sequencing resolution. ${ }^{2}$ The second one is the limitation of the current statistical models that try to relate genotypes to phenotype without accounting for the effect of combination of genes, or epistasis. ${ }^{3}$ These two reasons are believed to be a key to filling the "missing heritability" between genotypes and phenotypes. ${ }^{2}$

The word "epistasis" has been known for 100 years, ${ }^{4}$ and nowadays being broadly recognized as a key to elucidate complex human traits such as diabetes, cancer, obesity, and coronary heart disease. ${ }^{5,6}$ Detection of epistasis has attracted interests of numerous researchers, and many methods have been developed for this purpose, including MDR, ${ }^{7}$ PLINK, ${ }^{8}$ BOOST, ${ }^{3}$ GWIS, ${ }^{9}$ Fastanova $^{10}$ and so forth. These methods can perform single gene analysis in genomic scale, and some of them can handle even gene-gene interaction analysis in genomic scale. However, it is still hard for the current state-of-the-art approaches to detect interactions higher than twoways in genomic scale. Nonetheless, if true underlying genetic model is purely epistatic with degree $k>2$, then association methods analyzing up to two-way interactions should fail. ${ }^{11}$

At the other extreme, there is a line of research based on kernel methods that attempt to incorporate effects of many-way interactions into account. ${ }^{12-17}$ One can employ different kinds of kernels for modeling different genetic models. ${ }^{18} \mathrm{~A}$ popular choice is Gaussian kernels with which one can implicitly incorporate interaction of all possible degrees. ${ }^{18}$ Indeed, experimental results in real data ${ }^{13,15}$ show the high prediction accuracy of Gaussian kernels compared to conventional linear additive model. It therefore suggests the existence of non-additive effects in the real genomic data. In spite of all these nice properties, a serious drawback of using Gaussian kernels is that one cannot access salient features, and identify the causal genes.

In this paper, we propose a method that lies in between the two extremes mentioned above. The target degree of interaction also lies between the two, namely, $\geq 3$, and this definition applies to the term high-order interaction throughout the paper. Our approach is based on kernel ridge regression, or best linear unbiased prediction (BLUP) in the context of mixed models. ${ }^{15,19}$ We employ polynomial kernels in combination with model selection technique to predict the maximum degree of interactions in given data.

It is worth emphasizing that true degree of epistasis is unknown in general, and it is hard to gauge to which extent those interactions dominate complex traits. However, once maximum degree of epistasis is identified and available, then performing complete search becomes much easier inside the restricted search space. Our 
approach is straightforward; given SNP data, we build polynomial kernels with desired number of degrees, then choose the one with the smallest test error.

We evaluated the performance of our method in simulated data with artificially generated phenotypes, in which we know the true degree of epistasis. Experimental results showed the ability of our approach in predicting the true degree of epistasis under certain conditions. In cases when sufficient number of samples with respect to the number of SNPs is not available, we propose to perform sliding window approach, that is, to partition the whole SNP set into subregions, and examine the degree of epistasis within each subregion. With this sliding window approach, we can guarantee sufficient sample/SNP ratio in each subregion. In simulation study, sliding window approach could successfully detect the subregion with five-way SNP interactions. We also performed PLINK two-way interaction analysis in the same data, and confirmed that interacting SNPs found by PLINK was a part of the interacting SNPs found by our method.

We applied our method to heterogeneous stock mice data from the Jackson Laboratory, and compared the proposed method with existing approaches GSCANDB and PLINK. We demonstrate the flexibility of our method that does not need to specify the degree of epistasis unlike GSCANDB or PLINK.

We characterize the contribution of the proposed approach below: (1) our method can identify the degree of very high-order interaction even if the main effect is very weak or does not exist, (2) linear running time with respect to the number of SNPs, and (3) visualization of predicted regions with confidence scores.

This paper is organized as follows. In Sec. 2, we elaborate on our model based on polynomial kernels and model selection techniques. We also mention relationships between terminology in machine learning and that in genetics. In Sec. 3, we describe experimental details including preparation of data and parameter settings in ridge regression and sliding window approach. Section 4 consists of two subsections showing experimental results in both simulated data and mice data. In both subsections, we performed comparison to existing methods, and clarified pros and cons of the proposed method. Regarding the mice data, there are several reports on the causal SNPs, so we investigate to which extent consistency exits between already reported SNPs and those found by the proposed method. This paper concludes with discussion in Sec. 5 .

\section{Methods}

\subsection{Notation}

Given $n$ samples and $p$ SNPs, a widely used representation is to store them in an $n \times p$ matrix, in which each row corresponds to each sample, and each column corresponds to the minor allele counts of each sample. For example, if one allele position consists of nucleotide $\mathrm{A}$ as a major allele and $\mathrm{T}$ as a minor allele, then three allele types $\{\mathrm{AA}, \mathrm{AT}, \mathrm{TT}\}$ are coded as $\{0,1,2\}$, respectively. This representation is 
convenient in building additive model of minor alleles, but not appropriate for building other models such as additive model of major alleles or interaction model. Since we are interested in building interaction model, we employ binary representation of the data in a matrix $\mathbf{X} \in\{0,1\}^{n \times 3 p}$, in which each column corresponds to presence or absence of one of the three allele types. This representation allows us to build additive model of any alleles and their interaction models. At a glance, this representation seems to require three times as large storage as the former one, but indeed requires less storage size by using a bit representation. For example, the former representation requires $n \times p \times 8$ bits if we store minor allele count in an integer type, whereas the latter representation requires $n \times 3 p \times 1$ bits. Not only that, counting the co-occurrence of the same allele types between two samples can be efficiently performed by calling logical AND operation (also known as hamming weight ${ }^{20}$ ). In our approach, we need to count co-occurring allele types many times, so we employ bit representation. Below, we set $P=3 p$ for notational convenience.

\subsection{Linear regression, kernel ridge regression and connection to $G W A$ study}

Suppose we have SNP data in an $n \times P$ design matrix $\mathbf{X}$, and qualitative response $\boldsymbol{y} \in \mathcal{R}^{n}$ is available and centered. We begin with a standard linear model of the following form;

$$
f(\boldsymbol{x})=\sum_{j=1}^{P} \beta_{j} \boldsymbol{x}_{j},
$$

where $\boldsymbol{x}_{j}$ stands for the $j$ th variable of a test sample, and $\beta_{j}$ is the corresponding coefficient to be learned. We can determine $\boldsymbol{\beta}$ in such a way that sum of squares cost function

$$
c(f, \boldsymbol{x}, y)=\sum_{i=1}^{n}\left(f\left(\boldsymbol{x}_{i}\right)-y_{i}\right)^{2}
$$

is minimized over $n$ training data points. Solving this least squares problem typically leads to over fitting, so regularization is usually introduced. ${ }^{21}$ If we employ two-norm regularization on the coefficient vector $\boldsymbol{\beta}$ such that $\Omega(\boldsymbol{\beta})=\frac{\lambda}{2}\|\boldsymbol{\beta}\|_{2}^{2}$, then the problem is rephrased as

$$
\min c(f, \boldsymbol{x}, y)+\Omega(\boldsymbol{\beta}) .
$$

According to representer theorem, ${ }^{22}$ it can be shown that solution to the above problem admits not only Eq. (1), but also the following form;

$$
f(\boldsymbol{x})=\sum_{i=1}^{n} \alpha_{i} k\left(\boldsymbol{x}, \boldsymbol{x}^{\prime}\right),
$$


where $k(.,$.$) is a positive definite kernel, and \alpha_{i}$ is the coefficient for the $i$ th training data point. Suppose we have some feature mapping function $\phi($.$) , then kernel is$ a dot product of two mapped features;

$$
k\left(\boldsymbol{x}, \boldsymbol{x}^{\prime}\right)=\left\langle\phi(\boldsymbol{x}), \phi\left(\boldsymbol{x}^{\prime}\right)\right\rangle .
$$

We will see examples of mapping function $\phi($.$) below.$

Example 1. Consider the following case in which we have two samples $\boldsymbol{x}, \boldsymbol{x}^{\prime}$, each bearing two variables;

$$
\boldsymbol{x}=\left\{x_{1}, x_{2}\right\}, \quad \boldsymbol{x}^{\prime}=\left\{x_{1}^{\prime}, x_{2}^{\prime}\right\}
$$

Let us consider a simplest bijective function;

$$
\phi_{\text {linear }}:\left(x_{1}, x_{2}\right) \rightarrow\left(x_{1}, x_{2}\right),
$$

then the dot product of mapped samples $\boldsymbol{x}$ and $\boldsymbol{x}^{\prime}$ is

$$
\left\langle\phi_{\text {linear }}(\boldsymbol{x}), \phi_{\text {linear }}\left(\boldsymbol{x}^{\prime}\right)\right\rangle=x_{1} x_{1}^{\prime}+x_{2} x_{2}^{\prime}=\left\langle\boldsymbol{x}, \boldsymbol{x}^{\prime}\right\rangle .
$$

This form is known as linear kernel $k_{\text {linear }}\left(\boldsymbol{x}, \boldsymbol{x}^{\prime}\right)$. The least square solution obtained using linear kernel is equivalent to that obtained by using standard linear model in Eq. (1). The computation of linear kernel can be performed by one traversal of $P$-dimensional SNP space by calling a bitwise AND operation. Computation of allpairwise kernels $k(.,$.$) over n$ data points results in $n \times n$ kernel matrix $K \in \mathcal{R}^{n \times n}$, whose $(i, j)$ th entry stores the number of common allele types between $i$ th sample and $j$ th sample. In genetics, this matrix is known as kinship matrix by identity by state (IBS). In Ref. 23, weighted version of IBS kernel is proposed for amplifying the effect of rare variants for detecting causal variants that undergo "common diseaserare variants" hypothesis.

By devising a mapping function $\phi($.$) , we can take into consideration nonlinear$ effects such as gene-gene interactions. Let us explain examples of such function $\phi(.) \mathrm{s}$ below.

Example 2. Suppose we are given a monomial feature extractor function of degree 2:

$$
\phi_{\text {poly }}:\left(x_{1}, x_{2}\right) \rightarrow\left(x_{1} x_{1}, x_{2} x_{2}, x_{1} x_{2}, x_{2} x_{1}\right),
$$

then the dot product of mapped samples $\boldsymbol{x}$ and $\boldsymbol{x}^{\prime}$ is

$$
\left\langle\phi_{\text {poly }}(\boldsymbol{x}), \phi_{\text {poly }}\left(\boldsymbol{x}^{\prime}\right)\right\rangle=\left(x_{1} x_{1}^{\prime}\right)^{2}+\left(x_{2} x_{2}^{\prime}\right)^{2}+2\left(x_{1} x_{1}^{\prime}\right)\left(x_{2} x_{2}^{\prime}\right)=\left\langle\boldsymbol{x}, \boldsymbol{x}^{\prime}\right\rangle^{2} .
$$

This equation shows us two alternative ways to compute polynomial kernel. Either (1) to compute feature mapping (Eq. (6)) explicitly, then take their dot product, or (2) to compute dot product $\left\langle\boldsymbol{x}, \boldsymbol{x}^{\prime}\right\rangle$ in the input space, then squares it. In the latter way, one can omit the explicit computation of the feature map, which is 
attractive in our case, since we do not need to work with the feature space of size $P^{2}$, but instead with that of size $n^{2}$.

In general, two variables interaction model built by degree 2 polynomial kernel has the following form:

$$
\begin{aligned}
f(\boldsymbol{x}) & =\sum_{j=1}^{P} \sum_{j \leq k}^{P} \beta_{j, k}\left\langle\boldsymbol{x}, \boldsymbol{x}_{j}^{\prime}\right\rangle\left\langle\boldsymbol{x}, \boldsymbol{x}_{k}^{\prime}\right\rangle \\
& =\sum_{j=1}^{P} \beta_{j}\left\langle\boldsymbol{x}, \boldsymbol{x}_{j}^{\prime}\right\rangle^{2}+2 \sum_{j=1}^{P} \sum_{j<k}^{P} \beta_{j, k}\left\langle\boldsymbol{x}, \boldsymbol{x}_{j}^{\prime}\right\rangle\left\langle\boldsymbol{x}, \boldsymbol{x}_{k}^{\prime}\right\rangle .
\end{aligned}
$$

Equation (7) has $\frac{P(P+1)}{2}$ terms in total, so determining regression coefficients for all the terms is computationally intensive if $P$ is large. However, representer theorem holds not only for linear kernels, but also for nonlinear polynomial kernels. So if we employ polynomial kernel model with $n$ terms, then we can obtain the same regression error as the model with $\frac{P(P+1)}{2}$ terms. Likewise, degree $k$ polynomial kernel has the number of terms proportional to $P^{k}$, but we can work in the kernel feature space and compute the exact regression error independent of $P$. As we increase the degree of polynomial, the learned model starts overfitting to the training data, so we need to adjust regularization parameter $\lambda$ in Eq. (3) via cross-validation or generalized cross-validation. ${ }^{22}$ In experiments, we intensively use polynomial kernels with the degree from 1 to $k$. Specifically, the kernels we use are inhomogeneous ones $k_{\text {in }}\left(\boldsymbol{x}, \boldsymbol{x}^{\prime}\right)=\left(\left\langle x, x^{\prime}\right\rangle+1\right)^{k}$ instead of homogeneous ones $k_{\mathrm{ho}}\left(\boldsymbol{x}, \boldsymbol{x}^{\prime}\right)=\left(\left\langle x, x^{\prime}\right\rangle\right)^{k}$. Inhomogeneous polynomial kernels can detect interactions less than or equals to the specified number $k$, thereby more suitable for detecting the largest degree of interaction.

Next, we introduce Gaussian kernels, which are one of the most popular kernels due to their good performance in many applications including computational biology. ${ }^{24}$

Example 3. Consider the following Gaussian kernel between two samples $\boldsymbol{x}$ and $\boldsymbol{x}^{\prime}$ :

$$
k_{\text {gauss }}\left(\boldsymbol{x}, \boldsymbol{x}^{\prime}\right)=\exp \left(-\frac{\left\|\boldsymbol{x}-\boldsymbol{x}^{\prime}\right\|^{2}}{2 \sigma^{2}}\right) .
$$

Taylor expansion of the Gaussian kernel is:

$$
\begin{aligned}
k_{\text {gauss }}\left(\boldsymbol{x}, \boldsymbol{x}^{\prime}\right) & =\exp \left(\frac{\left\langle\boldsymbol{x}, \boldsymbol{x}^{\prime}\right\rangle}{\sigma^{2}}\right) \exp \left(-\frac{\|\boldsymbol{x}\|^{2}}{2 \sigma^{2}}\right) \exp \left(-\frac{\left\|\boldsymbol{x}^{\prime}\right\|^{2}}{2 \sigma^{2}}\right) \\
& =\sum_{k=0}^{\infty} \frac{1}{k !}\left(\frac{\left\langle\boldsymbol{x}, \boldsymbol{x}^{\prime}\right\rangle}{\sigma^{2}}\right)^{k} \exp \left(-\frac{\|\boldsymbol{x}\|^{2}}{2 \sigma^{2}}\right) \exp \left(-\frac{\left\|\boldsymbol{x}^{\prime}\right\|^{2}}{2 \sigma^{2}}\right),
\end{aligned}
$$

where the last equation is given by Taylor series of an exponential function: $\exp (x)=\sum_{k=0}^{\infty} \frac{x^{k}}{k !}$. Through this expression, we can see the Gaussian kernel as infinite dimensional union of polynomial kernels. The extent to which high-dimensional 
effect is incorporated is controlled by a $\sigma$ parameter. Smaller values of $\sigma$ emphasize the higher dimensional effects. ${ }^{12}$ In GWAS based on real and simulated data, Gaussian kernels are reported to outperform linear kernels, ${ }^{12,13,15}$ which suggests the importance of non-additive effects in relating genotypes to phenotypes. Further study and application of Gaussian kernels to real genomic data is awaited. ${ }^{12}$ However, Gaussian kernels are too flexible in a sense that all the possible polynomial effects are incorporated. To this end, we cannot tell how many degrees of interaction is necessary in explaining given phenotype. Thereafter in this paper, we focus on the polynomial kernels with fixed degree, and aim at predicting the maximum interaction degree within given data. Once the degree of polynomial is identified and available, then we can perform complete search much easily in the restricted search space.

\section{Experimental Procedure}

In this section, we first explain experimental settings of ridge regression. Then, we describe experimental procedure in simulated data. In simulated data, the true degree of epistasis is available, so we can evaluate the effectiveness of the proposed approach. On the other hand in real data, we do not know the true degree of epistasis, therefore we propose to apply sliding window approach.

\subsection{Ridge regression settings}

In comparing different degrees of polynomial kernels by regression performance, we employed four-fold cross-validation. We randomly split the whole SNPs into training, validation and test set, and computed the regression coefficients (Eq. (4)) in training data. Regularization parameter $\lambda$ is selected in validation set, and the final error rate is reported using test set. We employed standard residual sum of squares as error rate, and regularization parameter was chosen from $\{0.001,0.01,0.1,1,10$, $100\}$. The degrees of polynomial kernels to be surveyed ranged from 1 to 10 , and the one with the minimum error rate is reported. We repeated the above procedure 10 times, in order to get averaged performance.

\subsection{Simulation}

The simulation data are generated using gs version $2.0 .^{25, \mathrm{a}}$ It takes genotype/ haplotype data and a parameter file as inputs, and generates phenotype data based on either block method or extension method. Available parameters include the switching of single-locus/two-locus diseases model, maximum degree of epistasis, population prevalence and genotype relative risks. In $g s$, phenotypes are computed by logarithmic transformation of a random number, whose mean is reciprocal of a risk parameter. This approach is similar to the one used in genetics analysis

a http://engr.case.edu/li_jing/gs.html. 
workshop $15 .{ }^{26}$ We chose extension method, and used haplotype data in human Hapmap project. ${ }^{\mathrm{b}}$ We set minor allele frequency (MAF) and risk to 0.5 and 100 , respectively.

We evaluate the performance of the proposed method with respect to (1) increase in the number of SNPs and (2) increase in the number of samples.

So far, we have assumed that the degree of epistasis is always identifiable, independent of the sample/SNP ratio, however, as we see in the next section, accuracy of the proposed method depends heavily on the sample/SNP ratio, and the sensitivity degrades if the number of SNPs exceeds the number samples. For cases when sufficient sample/SNP ratio is not available, we propose to perform sliding window approach over chromosomal subregions for ensuring sufficiently large sample/SNP ratio in each subregion. In simulation, we prepared a sequence of random SNPs, and inserted five interacting SNPs by using the $g s$ software. We applied the sliding window approach with window length set to 100 SNPs and shifted by the increments of 25 SNPs. Note that we have overlaps in the neighboring windows, since it turned out to decrease prediction variance in experiments.

\subsection{Mouse inbred population}

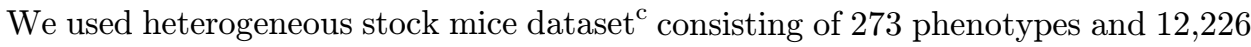
SNPs of at most 1940 mice. The mice were derived from eight inbred progenitor strains (A/J, AKR/J, BALB/cJ, C3H/HeJ, C57BL/6J, CBA/2J, DBA/2J and LP/J). The phenotype data set includes three common diseases (anxiety, type 2 diabetes and asthma), biochemistry, haematology and immunology profiles of each mouse. The number of SNPs in each chromosome is listed in Table 1 in supplementary material. In the real data, we do not know the true degree of epistasis, so we set the candidate degrees of epistasis to a wider range from 1 to 20. Other settings in ridge regression are same as those in the previous subsection.

We initially run cross-validation with different degrees of polynomial kernels over each chromosome, and chose the chromosome with the minimum error rate. Then, we performed sliding window approach on the selected chromosome with window length set to $100 \mathrm{SNPs}$, and shifted by $25 \mathrm{SNPs}$. In order to view the results from different resolution, we also performed the same analysis with window length and shift size set to 10 SNPs and single SNP, respectively.

Since we may have potentially too many causal genes interacting to each other, we define confidence score of a subregion by the minimum error rate achieved through different degrees of polynomial kernels. In order to make comparisons to existing tools, the above confidence score is converted in to $p$-values by performing one-sample $t$-test between a score from a specific region and scores from all the regions.

\footnotetext{
${ }^{b}$ http://hapmap.ncbi.nlm.nih.gov/downloads/index/html. In particular, we selected JPT and CHB haplotype data.

${ }^{\mathrm{c}}$ http://mus.well.ox.ac.uk/.
} 


\subsection{GSCANDB and PLINK}

In order to evaluate the proposed approach, we perform comparison to existing software; GSCANDB and PLINK. ${ }^{8}$ GSCANDB of Wellcome Trust Centre for Human Genetics ${ }^{\mathrm{d}}$ carries out single-locus analysis, in which variable selection is performed in a forward stepwise fashion, and $p$-value is computed by bootstrap aggregation. PLINK has an ability to conduct SNP $\times$ SNP epistasis analysis in its epistasis function, where $p$-value is computed by analysis of deviance (ANODEV). Note however that neither GSCANDB or PLINK considers interactions higher than two-ways, unlike the proposed method. We plot $p$-values of these methods together with $p$-values by our method.

\section{Results}

\subsection{Simulated data}

\subsubsection{Fixed window}

Figure 1(a) shows prediction accuracy in simulation data with respect to change in the number of SNPs while fixing the number of samples to 1000. Each curve shows

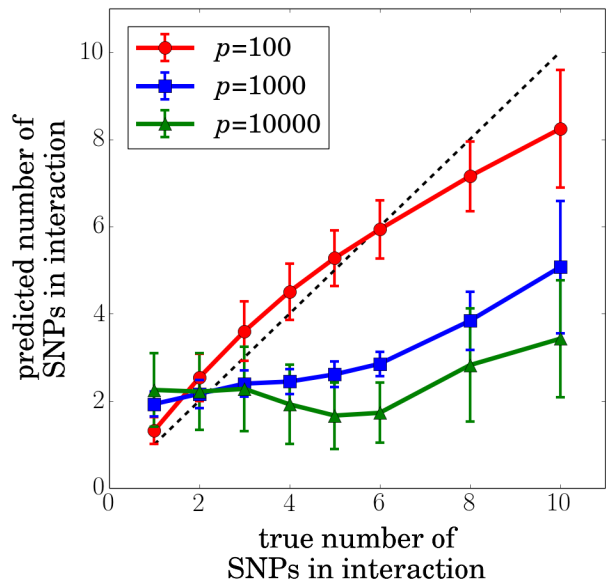

(a)

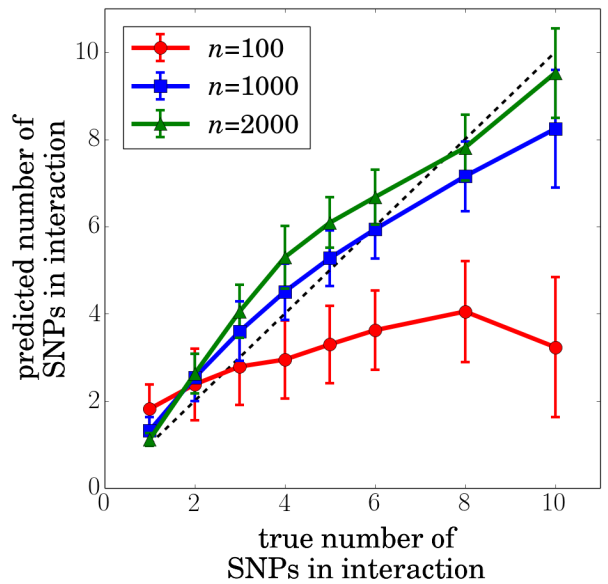

(b)

Fig. 1. Prediction accuracies with respect to increase in the number of SNPs and the number of individuals. The horizontal axis shows true number of SNPs in interaction, and the vertical axis shows predicted number of SNPs in interaction. Each curve shows prediction results with different parameters, and curves lying closer to the dotted line along the diagonal displays higher prediction accuracies. (a) The number of samples $(n)$ is set to 1000, and the number of SNPs $(p)$ is set to either 100 (red line, diamond marker), 1000 (blue line, square marker) or 10,000 (green line, triangle marker). (b) The number of SNPs $(p)$ is set to 100, and the number of samples $(n)$ is set to either 100 (red line, diamond marker), 1000 (blue line, square marker) or 2000 (green line, triangle marker).

d WTCHG, http://mus.well.ox.ac.uk/gscandb. 
prediction results with different parameters, and curves lying closer to the dotted line along the diagonal display higher prediction accuracies. We can observe that $p=100$ (red diamonds in Fig. 1(a)) was successful in predicting the degree of epistasis up to 6 , but starts under-estimating when the true degree is more than 6 . The reason for under-estimation in high degrees would be due to inclusion relationships among models. For example, if the true degree is 8 , but most of the variance in the phenotype would be explained by the model of degree 6 , then degree 6 is likely to be chosen as the model that explains data well. Curves corresponding to $p=1000$ (blue boxes in Fig. 1(a)) and $p=10,000$ (green triangle in Fig. 1(a)) always showed degraded prediction accuracies compared to that of $p=100$, which suggests the importance of sample/SNP ratio in our approach.

Figure 1(b) shows prediction accuracy in simulation data with respect to change in the number of samples while fixing the number of SNPs to 100. Predicted degree of epistasis was close to the true degree of epistasis when the number of samples is more than or equal to 1000 (lines with blue boxes and green triangles in Fig. 1(b)). This result again corroborates the importance of sample/SNP ratio, and shows the identifiably of high-order interactions.

\subsubsection{Sliding window approach}

Figure 2(left-top) shows regions predicted to harbor causal genes by sliding window approach. Window length was set to 100 SNPs, and shifted in the increments of 25 SNPs. We can identify the region with causal SNPs as a bump corresponding to small test set errors. By looking at the same horizontal region in box-plot in Fig. 2(leftmiddle), we can check the predicted degree of epistasis as between 3 and 4 , which was close to the true degree 5 . Note also the small prediction variance in those regions. On the other hand, we observed large prediction variance in regions outside of the ground truth region. We converted these observations into $p$-values and displayed in Manhattan plot in Fig. 2(left-bottom). It shows $p$-values by "test set errors" by our method (green solid line), together with $p$-values by PLINK epistasis function. A pair of SNPs with the smallest $p$-value found by PLINK are marked as red circles, and the other top 20 SNP pairs found by PLINK are plotted as black boxes. We can observe that all the five ground truth SNPs were correctly detected with high scores within top-20 SNP pairs. However, due to the limitation of PLINK, relationships between those high scoring SNPs was unclear. In contrast, our method detected chromosomal subregions containing five ground truth SNPs correctly (solid green curve in Fig. 2 (left-bottom)), and predicted the degree of epistasis as close to the true degree 5 . A limitation of our approach is that interacting SNPs must be located inside the sliding window. It is worth mentioning that there exists many ways to define SNP partitioning, for example, coding, intornic, inter-genic SNPs; MHC/non-MHC; sets of eQTL SNPs, ${ }^{27}$ and our approach is applicable to any of those SNP partitioning. Biologically meaningful partitioning can potentially put distant but interacting SNPs within the same window. 


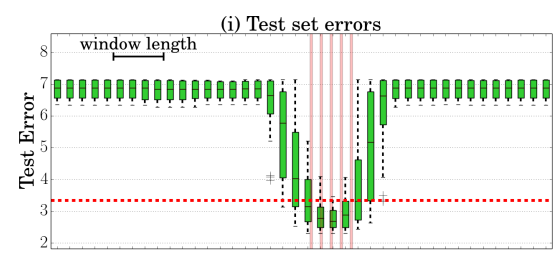

(ii) Predicted degree of epistasis
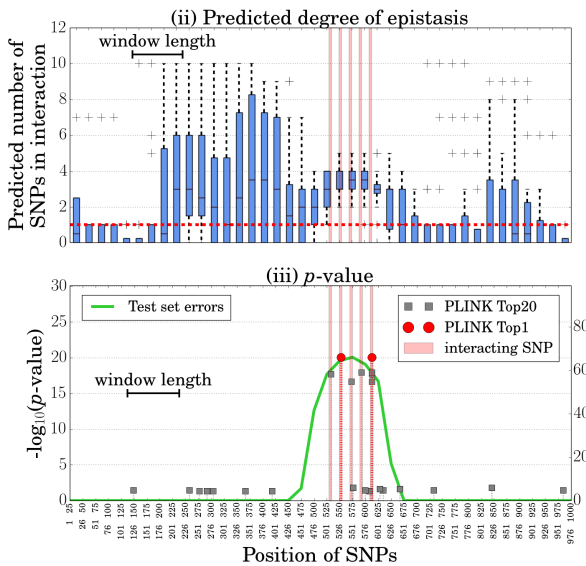

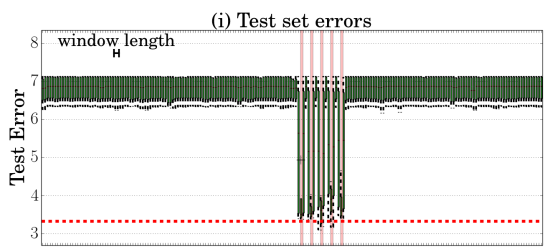

(ii) Predicted degree of epistasis
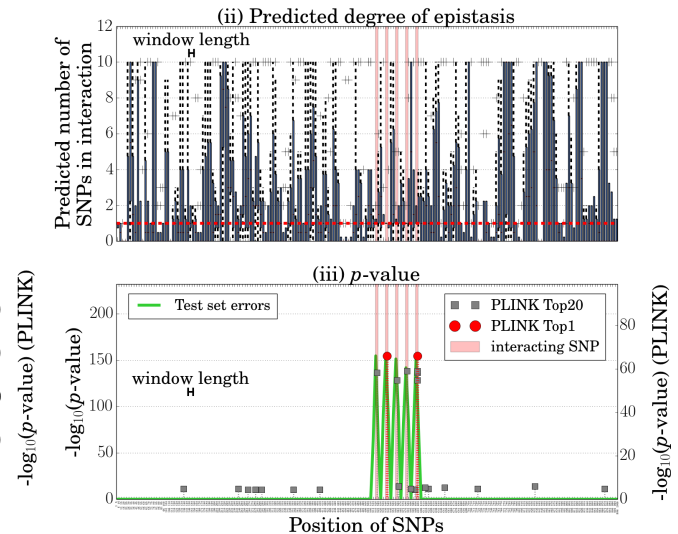

Fig. 2. Predicted location of five interacting SNPs in simulated data (marked by red vertical bars) by sliding window approach. The left column shows prediction results with window length set to 100 SNPs and shift size set to 25 SNPs. The right column shows prediction results with window length set to 10 SNPs and shift size set to 5 SNPs. (Top) Box-plot of minimum test set errors achieved by ridge regression in each subregion. Horizontal red dotted line shows mean test error of the all regions, and green boxes lying below the red dotted line correspond to predicted regions. (Middle) Box-plot of predicted degree of epistasis in each sliding window. Horizontal red dotted line shows mean predicted degree of all the regions. Note the small prediction variance in the region containing true interacting SNPs, but large prediction variance outside of the true region. (Bottom) Manhattan plot. Horizontal axis shows the positions of SNPs, and vertical axis shows- $\log 10(\mathrm{p})$ by our method (left-axis) and that by PLINK (right-axis). Solid green line corresponds to $p$-values by test set errors. For PLINK, the best SNP-SNP interaction pair is marked by red circles, and the other top 20 SNP pairs are plotted by black boxes.

Next, we changed window length and shift length to 10 and 5, respectively, and performed the same analysis over the same region. In Fig. 2 (right-top), we can observe that subregions containing ground truth SNPs are correctly identified. However, they were not detected as interacting to each other, but as five independent peaks (Fig. 2 (right-bottom)) due to the the shortage in window length. Although ground truth SNPs were not detected as interacting, we could successfully narrow down the position of the ground truth SNPs better than those by using a wider window size. These results altogether suggest the usefulness of using different window length for obtaining in-depth information about the positions and degree of interaction.

\subsection{Heterogeneous stock mice data}

We applied our method to various phenotypes in heterogeneous stock mice. We focus on "fear conditioning time freezing cue", but results in other phenotypes are 
available in supplementary material. We also clarify the meaning of "test set error" used in this subsection, since the ground truth SNPs are unavailable in real-world data. In each cross-validation fold, we first train several ridge regression models with various degrees of polynomials from input SNPs and phenotype values, then measure their errors in left-out test set, and finally report the smallest one as the "test set error". In cross-validation settings, they are averaged over different folds to one "test set error".

\subsubsection{Fear conditioning time freezing cue results}

Following the procedure in simulated data, we employed a strategy to narrow down the window size gradually. Figure 3(left-top) shows chromosome-wise prediction results. Box-plot of test set errors suggests existence of causal SNPs in chromosome 15 (red zone), so we focused on chromosome 15, and adopted sliding window approach with window length set to 100 SNPs, and shifted by 25 SNPs. To this end, we found $68.0-103.0 \mathrm{Mbp}$ region (red zone in Fig. 3 (right-top)) as a candidate region that harbors causal SNPs. Therefore we performed further in-depth analysis by narrowing down the sliding window length to 10 SNPs, and shifted by every single SNP. Figure 3 (bottom) shows prediction results in a region from 68.0 to 103.0 Mbp in chromosome 15. Test set errors were significantly small in a region from rs3694472 to rs6287587 (from 87.8 to $93.6 \mathrm{Mbp}$, red zone). Our method showed small test set errors together with small prediction variance, suggesting the existence of causal SNPs in this region. Results from GSCANDB (dotted orange line) and PLINK (red circles and black boxes) also showed consistent results (Fig. 3 (bottom)). Moreover, our analysis predicted the degree of epistasis as between 2 and 5 , so it is likely that more than two SNPs are interacting to each other in the selected region.

\subsubsection{Alkaline phosphatase ALP results}

We also elaborate on results in alkaline phosphatase (ALP) phenotype. Figure 3 in supplementary material shows $117-155 \mathrm{Mbp}$ region in chromosome 4 , which is selected by the same procedure as for "fear conditioning time freezing cue" phenotype. The purple bar shows the position of the known causal SNP (rs4224864) located in the coding region of Akp2 gene. ${ }^{28}$ The region containing this SNP was correctly detected by our method (solid green line) as well as by GSCANDB (dotted orange line). The shape of the peaks found by our method and GSCANDB were similar to each other. Considering that GSCANDB is a single-locus method, and our method detected the degree of epistasis as one, then it is likely that there are no more causal genes in the neighborhood. PLINK have failed in detecting the true regions, but this may be due to mis-specification of the PLINK option, namely that we forced twoway interaction mode and did not choose single-locus mode. However, this observation suggests the use of our method prior to PLINK in order to correctly specify the PLINK option. 


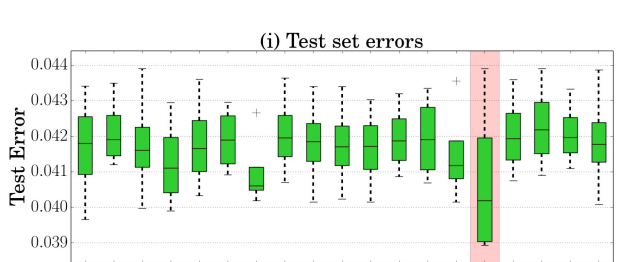

(ii) Predicted degree of epistasis

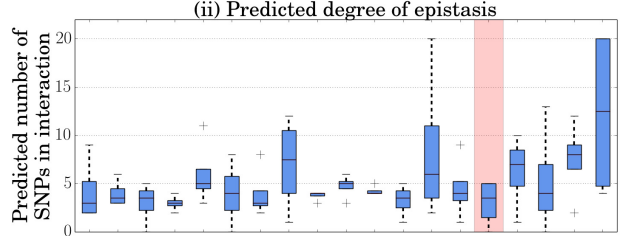

(iii) $p$-value

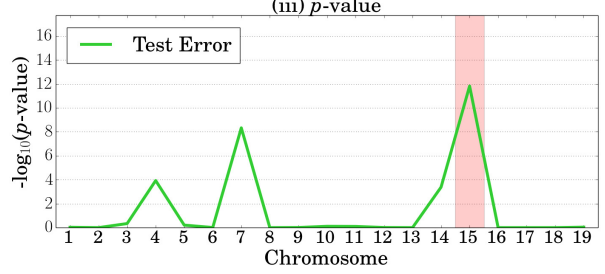

(i) Test set errors

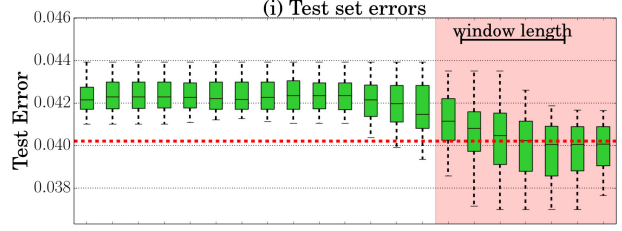

(ii) Predicted degree of epistasis

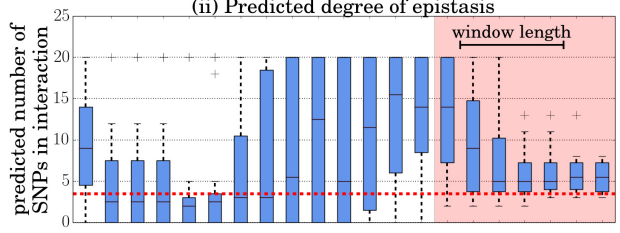

(iii) $p$-value

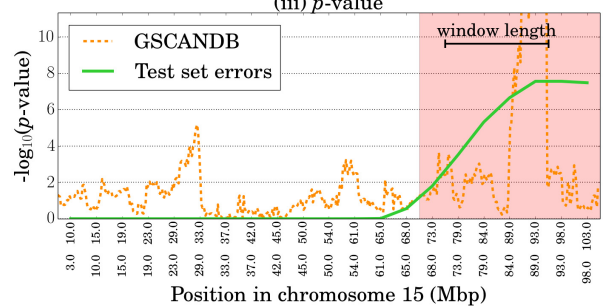

(i) Test set errors

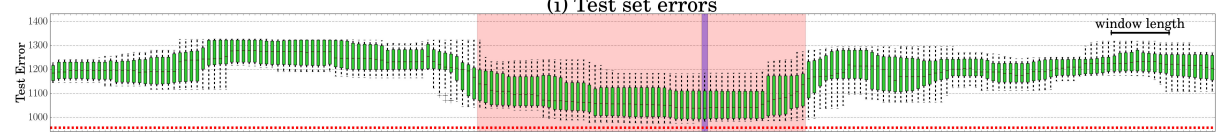

(ii) Predicted degree of epistasis

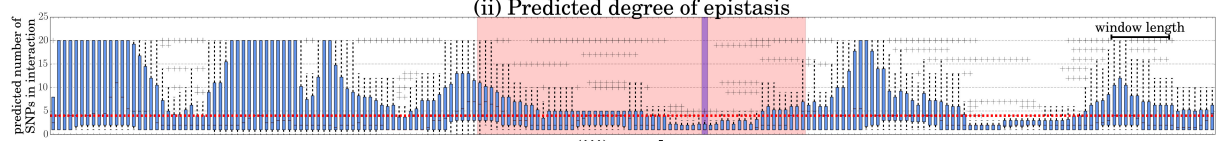

(iii) $p$-value

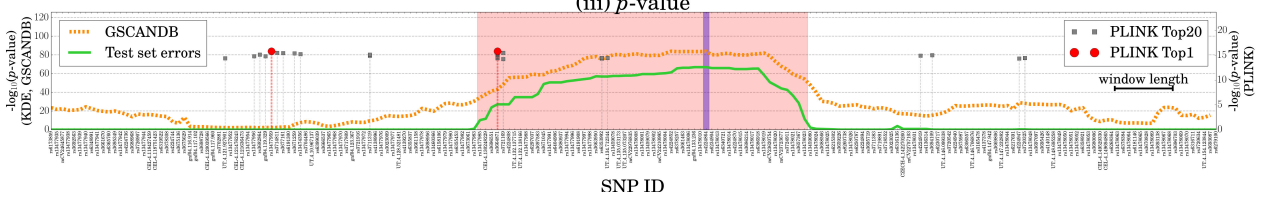

Fig. 3. Predicted location of causal SNPs for "fear conditioning time freezing cue" in different resolutions. (Top left) chromosome-wise prediction results. (Top right) prediction result inside chromosome 15 ( window size $=100$, shift $=10)$. (bottom) detailed prediction results over $68.0-103.0 \mathrm{Mbp}$ region in chromosome 15 (window size $=10$, shift size $=1$ ).

\section{Conclusion and Discussion}

In this paper, we proposed a kernel ridge regression-based approach for predicting the degree of epistasis. With the help of polynomial kernels and model selection technique, feasibility of the raised problem was demonstrated in simulated data. It was also shown that the use of sliding window is effective for cases in which large number of samples is not available. In comparison to SNP-SNP interaction detection 
method PLINK, the proposed method gives us richer information, as was shown in our experiments in simulated and mice data.

However, much work is left both in theory and practice. The two main issues are (1) computation of $p$-values and (2) determination of effective parameters (sample/ SNP ratio, window length, shift size) in real data.

(1) $P$-values: We employed one sample $t$-test between a score from a specific region and scores from all regions. This procedure implicitly assumes that all but the region under observation are drawn from the null distribution, which is unrealistic. A better alternative is performing permutation test by shuffling the phenotype in each window. However, performing permutation test is computationally too extensive with our current implementation, so acceleration of the algorithm is necessary beforehand. One such idea is to update QR decomposition when solving sequential ridge regression by making use of overlaps between neighboring windows.

(2) Effective parameters: In heterogeneous stock mice data, parameters to the algorithm such as sample/SNP ratio, window length and shift size were determined only empirically. It is generally hard to know those parameters in advance, and left remained as an open problem.

\section{Acknowledgment}

This work was supported by JSPS KAKENHI under Grant No. 25700004.

\section{References}

1. Makowsky R, Pajewski NM, Klimentidis YC, Vazquez AI, Duarte CW, Allison DB, de los Campos G, Beyond missing heritability: Prediction of complex traits, PLoS Genet. 7(4):e1002051, 2011.

2. Yang J, Benyamin B, McEvoy BP, Gordon S, Henders AK, Nyholt DR, Madden PA, Heath AC, Matin NG, Montgomery GW, Goddard ME, Visscher PM, Common SNPs explain a large proportion of the heritability for human height, Nat. Genet. 42:565-571, 2010.

3. Wan X, Yang C, Xue H, Fan X, Tang NLS, Yu W, Boost: A fast approach to detecting gene-gene interactions in genome-wide case-control studies, Am. J. Hum. Genet. 87:325$340,2010$.

4. Bateson W, Mendel G, Mendel's Principle of Heredity. Cambridge University Press, Cambridge, 1909.

5. Cordell HJ, Detecting gene-gene interactions that underlie human diseases, Nat. Rev. Genet. 10:392-404, 2009.

6. Nelson MR, Kardia SL, Ferell RE, Sing CF, A combinatorial partitioning method to idenity multilocus genotypic partitions that predict quantitative trait variation, Genome Res. 11:458-470, 201.

7. Ritchie MD, Hahn LW, Roodi N, Bailey LR, Dupont WD, Parl FF, Moore $\mathrm{JH}$, Multifactor-dimensionality reduction reveals high-order interactions among estrogen-metabolism genes in sporadic breast cancer, Am. J. Hum. Genet. 69:138-147, 2001 . 
8. Purcell S, Neale B, Brown KT, Thomas L, Ferreira MAR, Bender D, Maller J, Sklar P, de Bakker PIW, Daly MJ, Sham PC, Plink: A too set for whole-genome association and population-based linkage analysis, Am. J. Hum. Genet. 81:559-575, 2007.

9. Goudey B, Rawlinson D, Wang Q, Shi F, Ferra H, Campbell RM, Stern L, Inouye MT, Ong CS, Kowalczyk A, GWIS-model-free, fast and exhaustive search for epistatic interactions in case-control GWAS, BMC Genomics 14(Suppl3):S10, 2013.

10. Zhan X, Zou F, Wang W, Fastanova: An efficient algorithm for genome-wide association study, Proc. Int. Conf. Knowledge Discovery and Data Mining (KDD2008), pp. 821-829, 2008.

11. Culverhouse R, Suzrez BK, Lin J, Reich T, A perspective on epistasis: Limits of models displaying no main effect, Am. J. Hum. Genet. 70:461-471, 2002.

12. Liu D, Lin X, Ghosh D, Semiparametric regression of multidimensional genetic pathway data: Least-squares kernel machines and linear mixed models, Biometrics 63(4):1079-1088, 2007.

13. Gonzalez-Recio O, Gianola D, Long N, Weigel KA, Rosa GJM, Avendano S, Nonparametric methods for incorporating genomic information into genetic evaluations: An application to mortality in broilers, Genetics 178:2305-2313, 2008.

14. Gianola D, van Kaam JBCHM, Reproducing kernel hilbert spaces regression methods for genomic assisted prediction of quantitative traits, Genetics 178:2289-2303, 2008.

15. Endelman JB, Ridge regression and other kernels for genomic selection with $\mathrm{r}$ package rrBLUP, Plant Genome 4:250-255, 2011.

16. Shaoyu L, Cui Y, Gene-centric gene-gene interaction: A model-based kernel machine method, Ann. Stat. 6(3):1134-1161, 2012.

17. Lippert C, Xiang J, Horta D, Widmar C, Kadie C, Hackerman D, Listgarten J, Greater power and computational efficiency for kernel-based association testing of sets of genetic variants, Bioinformatics 30(22):3206-3214, 2014.

18. Schölkopf B, Smola AJ, Learning with Kernels: Support Vector Machines, Regularization, Optimization, and Beyond, MIT Press, Massachusetts, 2002.

19. Whittaker JC, Thompson R, Denham MC, Marker-assisted selection using ridge regression, Genet. Res. Camb. 75:249-252, 2000.

20. Knuth DE, The Art of Computer Programming, Bitwise Tricks $\& 5$ Techniques; Binary Decision Diagrams, Vol. 4, Fascicle 1, Addison Wesley Professional, Boston, 2009.

21. Hastie T, Tibshirani R, Friedman J, The Elements of Statistical Learning: Data Mining, Inference, and Prediction, Springer, New York, US, 2009.

22. Wahba G, Spline Models for Observational Data, CBMS-NSF Regional Conference Series in Applied Mathematics, Vol. 59, SIAM, Philadelphia, 1990.

23. Wu MC, Lee S, Cai T, Li Y, Boehnke M, Lin X, Rare-variant association testing for sequencing data with sequence kernel association test, Am. J. Hum. Genet. 89:82-93, 2011.

24. Schølkopf B, Tsuda K, Vert JP, Kernel Methods in Computational Biology, MIT Press, Massachusetts, US, 2004.

25. Chen Y, Li J, Generation of synthetic data and experimental designs in evaluating interactions for association studies, J. Bioinform. Comput. Biol. 10(1):1240005, 2012.

26. Miller MB, Lind GR, Li N, Jang SY, Genetic analysis workshop 15: Simulation of a complex genetic model for rheumatoid arthritis in nuclear families including a dense snp map with linkage disequilibrium between marker loci and trait loci, BMC Proc. 1(Suppl 1): S4, 2007.

27. Speed D, Balding DJ, Multiblup: Improved SNP-based prediction for complex traits, Genome Res. 24(9):1550-1557, 2014. 
28. Foreman JE, Blizard DA, Gerhard G, Mack HA, Lang DH, van Nimwegen KL, Vogler GP, Stout JT, Shihabi ZK, Griffith JW, Lakoski JM, McClearn GE, Vandenbergh DJ, Serum alkaline phosphatase activity is regulated by a chromosomal region containing the alkaline phosphatase 2 gene (akp2) in c57bl/6j and dba/2j mice, Physiol. Genomics 23:295-303, 2005.
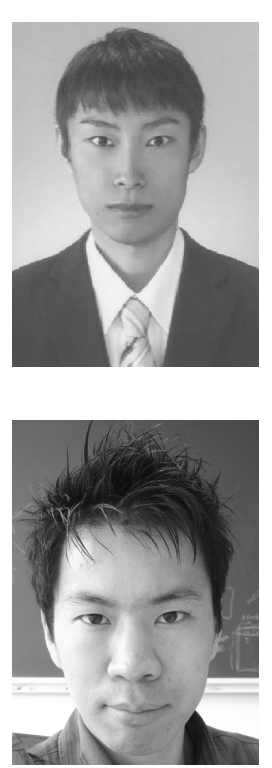

Kento Kodama received his Diploma and Master's degree, both in computer science and information technology, from Kyushu Institute of Technology (KIT), Fukuoka, Japan, in 2013 and 2015, respectively. He is currently in the Department of System Engineering, EM Systems company.

Hiroto Saigo received his B.E. degree from Sophia University in 2001. He obtained his Masters degree in Informatics from Kyoto University in 2003 and became a visiting student at the University of California Irvine until 2004. He received his Ph.D. from Kyoto University in 2006. He is currently an Associate Professor in the School of Information Science and Electrical Engineering, Kyushu University. His research interest is mainly in the developing methods for analyzing biological data, by utilizing statistics, data mining and machine learning. 